\title{
GENERAL DECAY OF SOLUTION FOR COUPLED SYSTEM OF VISCOELASTIC WAVE EQUATIONS OF KIRCHHOFF TYPE WITH DENSITY IN $\mathbb{R}^{n}$
}

\author{
Abbes Benaissa, Abderrahmane Beniani and Khaled Zennir
}

\begin{abstract}
A system of viscoelastic wave equations of Kirchhoff type is considered. For a wider class of relaxation functions, we use spaces weighted by the density function to establish a very general decay rate of the solution.
\end{abstract}

Keywords: Lyapunov function; Viscoelastic; Kirchhoff type; Density; Decay rate; Weighted spaces; Coupled system

\section{Introduction}

We consider the following system

$$
\left\{\begin{array}{l}
\left(\left|u^{\prime}\right|^{q-2} u^{\prime}\right)^{\prime}-\phi(x)\left(M\left(\left\|\nabla_{x} u\right\|_{2}^{2}\right) \Delta_{x} u-\int_{0}^{t} g_{1}(t-s) \Delta_{x} u(s) d s\right)+\alpha v=0 \\
\left(\left|v^{\prime}\right|^{q-2} v^{\prime}\right)^{\prime}-\phi(x)\left(M\left(\left\|\nabla_{x} v\right\|_{2}^{2}\right) \Delta_{x} v-\int_{0}^{t} g_{2}(t-s) \Delta_{x} v(s) d s\right)+\alpha u=0
\end{array}\right.
$$

where $x \in \mathbb{R}^{n}, \alpha, t>0, q, n \geq 2$ and $M$ is a positive $C^{1}$ function satisfying for $s \geq 0, m_{0}>0, m_{1} \geq 0, \gamma \geq 1, M(s)=m_{0}+m_{1} s^{\gamma}$ and the scalar functions $g_{i}(s), i=1,2$ (the so-called relaxation kernel) are assumed to satisfy (A1).

The problem (1.1) is equipped by the following initial data.

$$
\begin{aligned}
& u(0, x)=u_{0}(x) \in \mathcal{H}\left(\mathbb{R}^{n}\right), \quad u^{\prime}(0, x)=u_{1}(x) \in L_{\rho}^{q}\left(\mathbb{R}^{n}\right), \\
& v(0, x)=v_{0}(x) \in \mathcal{H}\left(\mathbb{R}^{n}\right), \quad v^{\prime}(0, x)=v_{1}(x) \in L_{\rho}^{q}\left(\mathbb{R}^{n}\right),
\end{aligned}
$$

where the weighted spaces $\mathcal{H}$ is given in Definition 2.1 and the density function $(\phi(x))^{-1}=\rho(x)$ satisfies

$$
\rho: \mathbb{R}^{n} \rightarrow \mathbb{R}_{+}^{*}, \quad \rho(x) \in C^{0, \tilde{\gamma}}\left(\mathbb{R}^{n}\right)
$$

Received November 25, 2015; Accepted March 29, 2016

2010 Mathematics Subject Classification. Primary 35L05; Secondary 35L70, 35B05 
with $\tilde{\gamma} \in(0,1)$ and $\rho \in L^{s}\left(\mathbb{R}^{n}\right) \cap L^{\infty}\left(\mathbb{R}^{n}\right)$, where $s=\frac{2 n}{2 n-q n+2 q}$.

In this framework, (see [8], [9], [15], [16], [19], [21]), it is well known that for any initial data $u_{0}, v_{0} \in \mathcal{H}\left(\mathbb{R}^{n}\right)$ and $u_{1}, v_{1} \in L_{\rho}^{q}\left(\mathbb{R}^{n}\right)$, the problem (1.1)-(1.3) has a unique solution $(u, v) \in\left(C\left([0, T), \mathcal{H}\left(\mathbb{R}^{n}\right)\right)\right)^{2},\left(u^{\prime}, v^{\prime}\right) \in\left(C\left([0, T), L_{\rho}^{q}\left(\mathbb{R}^{n}\right)\right)\right)^{2}$, under the hypothesis $(A 1)-(A 2)$. The problem (1.1) is usually encountered in viscoelasticity in various areas of mathematical physics, it was first considered by Dafermos in [7], where the general decay was discussed. The problems related to (1.1) have attracted a great deal of attention in the last decades and numerous results appeared on the existence and long time behavior of solutions but their results are by now rather developed, especially in any space dimension.

This kind of system appears in the models of the nonlinear Kirchhoff-type. It is a generalization of a model introduced by Kirchhoff [13] as the equation in the case $n=1$ describes this type of problem as a small amplitude vibration of an elastic string. The original equation is:

$$
\rho h u_{t t}+\tau u_{t}=\left(P_{0}+\frac{E h}{2 L} \int_{0}^{L}\left|u_{x}(x, t)\right|^{2} d s\right) u_{x x}+f,
$$

where $0 \leq x \leq L$ and $t>0, u(x, t)$ is the lateral displacement at the space coordinate $x$ and the time $t, \rho$ the mass density, $h$ the cross-section area, $L$ the length, $P_{0}$ the initial axial tension, $\tau$ the resistance modulus, $E$ the Young modulus and $f$ the external force (for example, the action of gravity).

The motivation for our study is due to some results regarding viscoelastic wave equations of the Kirchhoff type in a bounded domain. The wave equation of the form

$$
u^{\prime \prime}-M\left(\left\|\nabla_{x} u\right\|_{2}^{2}\right) \Delta_{x} u+\int_{0}^{t} g(t-s) \Delta_{x} u(s) d s+h\left(u^{\prime}\right)=f(u), \quad x \in \Omega, t>0
$$

is a model to describe the motion of deformable solids as the hereditary effect is incorporated. Eq.(1.6) was studied by [20] and they proved the existence of weak solution for a large datum. Later, for a small datum and under some restrictions, global solutions and exponential decay to zero is shown in [17].

The work with weighted spaces was studied by many authors (see in this direction [5], [12], [18] and [22]). For the decay rate of solution for equations in $\mathbb{R}^{n}$, we quote the results by [2], [9], [10], [11], [16]. In [10], the authors showed that for compactly supported initial data and for an exponentially decaying relaxation function the decay of the energy of the solution to the linear Cauchy problem (1.1),(1.3) in one equation with $\alpha=0, q=2, \rho(x)=1, M \equiv 1$ is polynomial. The finite-speed propagation is used to compensate for the lack of Poincare's inequality. In case $\alpha=0, q=2, M \equiv 1$, in [9], the author looked into a linear Cauchy viscoelastic problem with density. His study included the exponential and polynomial rates, where he used the spaces weighted by density to compensate for the lack of Poincare's inequality. The same problem, treated in [9], was considered in [11], 
where they considered the Cauchy problem for the viscoelastic wave equation. Under suitable conditions on the initial data and the relaxation function, they prove a polynomial decay result of solutions. The conditions used on the relaxation function $g$ and its derivative $g^{\prime}$ are different from the usual ones.

The problem (1.1),(1.3) in case $\alpha=0, x \in \mathbb{R}^{n}$ with the relaxation function $g$ is a positive nonincreasing function, was considered as one equation in [21], where the author established a general decay rate result for relaxation functions satisfying the assumptions $(A 1)-(A 2)$. The main purpose of the present paper is to extend this result to a coupled system of linear equations.

We omit the space variable $x$ of $u(x, t), u^{\prime}(x, t)$ and, for simplicity reasons, denote $u(x, t)=u$ and $u^{\prime}(x, t)=u^{\prime}$, when no confusion arises. The constants $c$ used throughout this paper are positive generic constants which may be different, here $u^{\prime}=d u(t) / d t$ and $u^{\prime \prime}=d^{2} u(t) / d t^{2}$.

\section{Material, spaces and Assumptions}

First, we recall and make use the following assumptions on the functions $g_{i}, i=1,2$ as:

(A1) We assume that the functions $g_{i}: \mathbb{R}^{+} \longrightarrow \mathbb{R}^{+}$is of class $C^{1}$ satisfying:

$$
m_{0}-\overline{g_{i}}=l_{i}>0, \quad g_{i}(0)=g_{0 i}>0,
$$

where $\overline{g_{i}}=\int_{0}^{\infty} g_{i}(t) d t$.

(A2) There exists a positive function $H \in C^{1}\left(\mathbb{R}^{+}\right)$such that

$$
g_{i}^{\prime}(t)+H\left(g_{i}(t)\right) \leq 0, t \geq 0, \quad H(0)=0
$$

and $H$ is linear or strictly increasing and strictly convex $C^{2}$ function on $(0, r], 1>r$.

Remark 2.1. [16]

A) We can deduce that there exists $t_{1}>0$ large enough such that for $i=1,2$ :

1) $\forall t \geq t_{1}$ : We have $\lim _{s \rightarrow+\infty} g_{i}(s)=0$, which implies that $\lim _{s \rightarrow+\infty}-g_{i}^{\prime}(s)$ cannot be positive, so $\lim _{s \rightarrow+\infty}-g_{i}^{\prime}(s)=0$. Then $g_{i}\left(t_{1}\right)>0$ and

$$
\max \left\{g_{1}(s), g_{2}(s),-g_{1}^{\prime}(s),-g_{2}^{\prime}(s)\right\}<\min \left\{r, H(r), H_{0}(r)\right\},
$$

where $H_{0}(t)=H(D(t))$ provided that $D$ is a positive $C^{1}$ function, with $D(0)=0$, for which $H_{0}$ is a strictly increasing and strictly convex $C^{2}$ function on $(0, r]$ and

$$
\int_{0}^{+\infty} g_{i}(s) H_{0}\left(-g_{i}^{\prime}(s)\right) d s<+\infty .
$$

2) $\forall t \in\left[0, t_{1}\right]$ : As $g_{i}$ are nonincreasing, $g_{i}(0)>0$ and $g_{i}\left(t_{1}\right)>0$ then $g_{i}(t)>0$ and

$$
g_{i}(0) \geq g_{i}(t) \geq g_{i}\left(t_{1}\right)>0 .
$$


Therefore, since $H$ is a positive continuous function, then

$$
\begin{aligned}
& a \leq H\left(g_{1}(t)\right) \leq b \\
& c \leq H\left(g_{2}(t)\right) \leq d
\end{aligned}
$$

for some positive constants $a, b, c$ and $d$. Consequently,

$$
g_{i}^{\prime}(t) \leq-H\left(g_{i}(t)\right) \leq-k g_{i}(t), \quad k>0
$$

which gives

$$
g_{i}^{\prime}(t) \leq-k g_{i}(t), k>0
$$

B) Let $H_{0}^{*}$ be the convex conjugate of $H_{0}$ in the sense of Young (see [3], pages 61-64), then

$$
H_{0}^{*}(s)=s\left(H_{0}^{\prime}\right)^{-1}(s)-H_{0}\left[\left(H_{0}^{\prime}\right)^{-1}(s)\right], \quad s \in\left(0, H_{0}^{\prime}(r)\right)
$$

and satisfies the following Young's inequality

$$
A B \leq H_{0}^{*}(A)+H_{0}(B), \quad A \in\left(0, H_{0}^{\prime}(r)\right), B \in(0, r] .
$$

Definition 2.1. [[9], [18]] We define the function spaces of our problem and its norm as follows:

$$
\mathcal{H}\left(\mathbb{R}^{n}\right)=\left\{f \in L^{2 n /(n-2)}\left(\mathbb{R}^{n}\right): \nabla_{x} f \in\left(L^{2}\left(\mathbb{R}^{n}\right)\right)^{n}\right\}
$$

and the space $L_{\rho}^{2}\left(\mathbb{R}^{n}\right)$ to be the closure of $C_{0}^{\infty}\left(\mathbb{R}^{n}\right)$ functions with respect to the inner product

$$
(f, h)_{L_{\rho}^{2}\left(\mathbb{R}^{n}\right)}=\int_{\mathbb{R}^{n}} \rho f h d x .
$$

For $1<q<\infty$, if $f$ is a measurable function on $\mathbb{R}^{n}$, we define

$$
\|f\|_{L_{\rho}^{q}\left(\mathbb{R}^{n}\right)}=\left(\int_{\mathbb{R}^{n}} \rho|f|^{q} d x\right)^{1 / q}
$$

The space $L_{\rho}^{2}\left(\mathbb{R}^{n}\right)$ is a separable Hilbert space.

The following technical lemma will play an important role in the sequel.

Lemma 2.1. [6] (Lemma 1.1) For any two functions $h, v \in C^{1}(\mathbb{R})$ and $\theta \in[0,1]$ we have

$$
\begin{aligned}
v^{\prime}(t) \int_{0}^{t} h(t-s) v(s) d s & =-\frac{1}{2} \frac{d}{d t} \int_{0}^{t} h(t-s)|v(t)-v(s)|^{2} d s+\frac{1}{2} \frac{d}{d t}\left(\int_{0}^{t} h(s) d s\right)|v(t)|^{2} \\
& +\frac{1}{2} \int_{0}^{t} h^{\prime}(t-s)|v(t)-v(s)|^{2} d s-\frac{1}{2} h(t)|v(t)|^{2} .
\end{aligned}
$$


and

$$
\begin{aligned}
& \left|\int_{0}^{t} h(t-s)(v(t)-v(s)) d s\right|^{2} \\
& \quad \leq\left(\int_{0}^{t}|h(s)|^{2(1-\theta)} d s\right)\left(\int_{0}^{t}|h(t-s)|^{2 \theta}|v(t)-v(s)|^{2} d s\right)
\end{aligned}
$$

The energy of $(u, v)$ at time $t$ is defined by

$$
\begin{aligned}
E(t)= & \frac{(q-1)}{q}\left[\left\|u^{\prime}\right\|_{L_{\rho}^{q}\left(\mathbb{R}^{n}\right)}^{q}+\left\|v^{\prime}\right\|_{L_{\rho}^{q}\left(\mathbb{R}^{n}\right)}^{q}\right] \\
& +\frac{m_{1}}{2(\gamma+1)}\left[\left\|\nabla_{x} u\right\|_{2}^{2(\gamma+1)}+\left\|\nabla_{x} v\right\|_{2}^{2(\gamma+1)}\right] \\
& +\frac{1}{2}\left(m_{0}-\int_{0}^{t} g_{1}(s) d s\right)\left\|\nabla_{x} u\right\|_{2}^{2}+\frac{1}{2}\left(m_{0}-\int_{0}^{t} g_{2}(s) d s\right)\left\|\nabla_{x} v\right\|_{2}^{2} \\
+ & \frac{1}{2}\left(g_{1} \circ \nabla_{x} u\right)+\frac{1}{2}\left(g_{2} \circ \nabla_{x} v\right)+\alpha \int_{\mathbb{R}^{n}} \rho u v d x
\end{aligned}
$$

For $\alpha$ small enough we use Lemma 3.1 to deduce for $c>0$ that:

$$
\begin{aligned}
E(t) & \geq\left(1-c|\alpha|\|\rho\|_{L^{n / 2}}^{-1}\right) \frac{(q-1)}{q}\left[\left\|u^{\prime}\right\|_{L_{\rho}^{q}\left(\mathbb{R}^{n}\right)}^{q}+\left\|v^{\prime}\right\|_{L_{\rho}^{q}\left(\mathbb{R}^{n}\right)}^{q}\right] \\
& +\frac{m_{1}}{2(\gamma+1)}\left[\left\|\nabla_{x} u\right\|_{2}^{2(\gamma+1)}+\left\|\nabla_{x} v\right\|_{2}^{2(\gamma+1)}\right] \\
& +\frac{1}{2}\left(m_{0}-\int_{0}^{t} g_{1}(s) d s\right)\left\|\nabla_{x} u\right\|_{2}^{2}+\frac{1}{2}\left(m_{0}-\int_{0}^{t} g_{2}(s) d s\right)\left\|\nabla_{x} v\right\|_{2}^{2} \\
& +\frac{1}{2}\left(g_{1} \circ \nabla_{x} u\right)+\frac{1}{2}\left(g_{2} \circ \nabla_{x} v\right)
\end{aligned}
$$

and the following energy functional law holds:

$$
E^{\prime}(t) \leq \frac{1}{2}\left(g_{1}^{\prime} \circ \nabla_{x} u\right)(t)+\frac{1}{2}\left(g_{2}^{\prime} \circ \nabla_{x} v\right)(t), \quad \text { for all } \quad t \geq 0 .
$$

which means that our energy is uniformly bounded and decreasing along the trajectories. The following notation will be used throughout this paper

$$
\left(g_{i} \circ \nabla_{x} \psi\right)(t)=\int_{0}^{t} g_{i}(t-\tau)\left\|\nabla_{x} \psi(t)-\nabla_{x} \psi(\tau)\right\|_{2}^{2} d \tau, i=1,2,
$$

for $\psi(t) \in \mathcal{H}\left(\mathbb{R}^{n}\right), t \geq 0$.

We are now ready to state and prove our main results.

\section{Main results}

The next Lemma can be easily shown (see [12], Lemma 2.1). 
Lemma 3.1. Let $\rho$ satisfies (1.4), then for any $u \in \mathcal{H}\left(\mathbb{R}^{n}\right)$

$$
\|u\|_{L_{\rho}^{q}\left(\mathbb{R}^{n}\right)} \leq\|\rho\|_{L^{s}\left(\mathbb{R}^{n}\right)}\left\|\nabla_{x} u\right\|_{L^{2}\left(\mathbb{R}^{n}\right)}
$$

with $s=\frac{2 n}{2 n-q n+2 q}, 2 \leq q \leq \frac{2 n}{n-2}$

Our main result reads as follows.

Theorem 3.1. Let $\left(u_{0}, v_{0}\right) \in\left(\mathcal{H}\left(\mathbb{R}^{n}\right)\right)^{2},\left(u_{1}, v_{1}\right) \in\left(L_{\rho}^{q}\left(\mathbb{R}^{n}\right)\right)^{2}$ and suppose that $(A 1)-(A 2)$ hold. Then there exist positive constants $a, b, c, d$ such that the energy of the solution given by (1.1),(1.3) satisfies,

$$
E(t) \leq d H_{1}^{-1}(b t+c), \quad \text { for all } t \geq 0,
$$

where

$$
H_{1}(t)=\int_{t}^{1} \frac{1}{s H_{0}^{\prime}(a s)} d s
$$

To prove Theorem 3.1, let us define

$$
L(t)=\xi_{1} E(t)+\psi_{1}(t)+\xi_{2} \psi_{2}(t)
$$

for $\xi_{1}, \xi_{2}>1$. In order to obtain useful estimates, we prepare some functionals associated with the nature of our problem introduced in Lyapunov function $L$ as

$$
\psi_{1}(t)=\int_{\mathbb{R}^{n}} \rho(x)\left[u\left|u^{\prime}\right|^{q-2} u^{\prime}+v\left|v^{\prime}\right|^{q-2} v^{\prime}\right] d x,
$$

and the existence of the memory terms force us to introduce the next functional

$$
\begin{aligned}
\psi_{2}(t) & =-\int_{\mathbb{R}^{n}} \rho(x)\left|u^{\prime}\right|^{q-2} u^{\prime} \int_{0}^{t} g_{1}(t-s)(u(t)-u(s)) d s d x \\
& -\int_{\mathbb{R}^{n}} \rho(x)\left|v^{\prime}\right|^{q-2} v^{\prime} \int_{0}^{t} g_{2}(t-s)(v(t)-v(s)) d s d x .
\end{aligned}
$$

Lemma 3.2. Under the assumptions (A1) and (A2), the functional $\psi_{1}$ satisfies, along the solution to (1.1),(1.3)

$$
\begin{aligned}
\psi_{1}^{\prime}(t) \leq & \left(1-d \alpha \mid\|\rho\|_{L^{n / 2}}^{-1}\right)\left[\left\|u^{\prime}\right\|_{L_{\rho}^{q}\left(\mathbb{R}^{n}\right)}^{q}+\left\|v^{\prime}\right\|_{L_{\rho}^{q}\left(\mathbb{R}^{n}\right)}^{q}\right] \\
& +c_{1} m_{1}\left(\left\|\nabla_{x} u\right\|_{2}^{2(\gamma+1)}+\left\|\nabla_{x} v\right\|_{2}^{2(\gamma+1)}\right) \\
& +(\sigma-l)\left(\left\|\nabla_{x} u\right\|_{2}^{2}+\left\|\nabla_{x} v\right\|_{2}^{2}\right)+\frac{(1-l)}{4 \sigma}\left(\left(g_{1} \circ \nabla_{x} u\right)+\left(g_{2} \circ \nabla_{x} v\right)\right),
\end{aligned}
$$

where $l=\min \left\{l_{1}, l_{2}\right\}$. 
Proof. From (3.4), integrate over $\mathbb{R}^{n}$, we have

$$
\begin{aligned}
\psi_{1}^{\prime}(t) & =\int_{\mathbb{R}^{n}} \rho(x)\left|u^{\prime}\right|^{q} d x+\int_{\mathbb{R}^{n}} \rho(x) u\left(\left|u^{\prime}\right|^{q-2} u^{\prime}\right)^{\prime} d x \\
& +\int_{\mathbb{R}^{n}} \rho(x)\left|v^{\prime}\right|^{q} d x+\int_{\mathbb{R}^{n}} \rho(x) v\left(\left|v^{\prime}\right|^{q-2} v^{\prime}\right)^{\prime} d x \\
& =\int_{\mathbb{R}^{n}}\left(\rho(x)\left|u^{\prime}\right|^{q}+M\left(\left\|\nabla_{x} u\right\|_{2}^{2}\right) u \Delta_{x} u-u \int_{0}^{t} g_{1}(t-s) \Delta_{x} u(s, x) d s-\alpha u v\right) d x \\
& +\int_{\mathbb{R}^{n}}\left(\rho(x)\left|v^{\prime}\right|^{q}+M\left(\left\|\nabla_{x} v\right\|_{2}^{2}\right) v \Delta_{x} v-v \int_{0}^{t} g_{2}(t-s) \Delta_{x} v(s, x) d s-\alpha u v\right) d x \\
& \leq\left(1-c|\alpha|\|\rho\|_{L^{n / 2}}^{-1}\right)\left[\left\|u^{\prime}\right\|_{L_{\rho}^{q}\left(\mathbb{R}^{n}\right)}^{q}+\left\|v^{\prime}\right\|_{L_{\rho}^{q}\left(\mathbb{R}^{n}\right)}^{q}\right]+m_{1}\left(\left\|\nabla_{x} u\right\|_{2}^{2(\gamma+1)}+\left\|\nabla_{x} v\right\|_{2}^{2(\gamma+1)}\right) \\
& +\int_{\mathbb{R}^{n}} \nabla_{x} u \int_{0}^{t} g_{1}(t-s)\left(\nabla_{x} u(s)-\nabla_{x} u(t)\right) d s d x-l_{1}\left\|\nabla_{x} u\right\|_{2}^{2} \\
& +\int_{\mathbb{R}^{n}} \nabla_{x} v \int_{0}^{t} g_{2}(t-s)\left(\nabla_{x} v(s)-\nabla_{x} v(t)\right) d s d x-l_{2}\left\|\nabla_{x} v\right\|_{2}^{2} .
\end{aligned}
$$

Using Young's inequality and Lemma 2.1 for $\theta=1 / 2$, we obtain for $l=\min \left\{l_{1}, l_{2}\right\}$

$$
\begin{aligned}
\psi_{1}^{\prime}(t) & \leq\left(1-c|\alpha|\|\rho\|_{L^{n / 2}}^{-1}\right)\left[\left\|u^{\prime}\right\|_{L_{\rho}^{q}\left(\mathbb{R}^{n}\right)}^{q}+\left\|v^{\prime}\right\|_{L_{\rho}^{q}\left(\mathbb{R}^{n}\right)}^{q}\right]+m_{1}\left(\left\|\nabla_{x} u\right\|_{2}^{2(\gamma+1)}+\left\|\nabla_{x} v\right\|_{2}^{2(\gamma+1)}\right) \\
& -l_{1}\left\|\nabla_{x} u\right\|_{2}^{2}-l_{2}\left\|\nabla_{x} v\right\|_{2}^{2} \\
& +\sigma\left\|\nabla_{x} u\right\|_{2}^{2}+\frac{1}{4 \sigma} \int_{\mathbb{R}^{n}}\left(\int_{0}^{t} g_{1}(t-s)\left|\nabla_{x} u(s)-\nabla_{x} u(t)\right| d s\right)^{2} d x \\
& +\sigma\left\|\nabla_{x} v\right\|_{2}^{2}+\frac{1}{4 \sigma} \int_{\mathbb{R}^{n}}\left(\int_{0}^{t} g_{2}(t-s)\left|\nabla_{x} v(s)-\nabla_{x} v(t)\right| d s\right)^{2} d x \\
& \leq\left(1-c|\alpha|\|\rho\|_{L^{n / 2}}^{-1}\right)\left[\left\|u^{\prime}\right\|_{L_{\rho}^{q}\left(\mathbb{R}^{n}\right)}^{q}+\left\|v^{\prime}\right\|_{L_{\rho}^{q}\left(\mathbb{R}^{n}\right)}^{q}\right]+m_{1}\left(\left\|\nabla_{x} u\right\|_{2}^{2(\gamma+1)}+\left\|\nabla_{x} v\right\|_{2}^{2(\gamma+1)}\right) \\
& +(\sigma-l)\left(\left\|\nabla_{x} u\right\|_{2}^{2}+\left\|\nabla_{x} v\right\|_{2}^{2}\right)+\frac{(1-l)}{4 \sigma}\left(\left(g_{1} \circ \nabla_{x} u\right)+\left(g_{2} \circ \nabla_{x} v\right)\right) .
\end{aligned}
$$

Lemma 3.3. Under the assumptions (A1) and (A2), the functional $\psi_{2}$ satisfies, along the solution of $(1.1),(1.3)$, for any $\sigma \in\left(0, m_{0}\right)$

$$
\begin{aligned}
\psi_{2}^{\prime}(t) & \leq \sigma\left(1+\alpha\|\rho\|_{L^{n / 2}\left(\mathbb{R}^{n}\right)}^{2}\right)\left(\left\|\nabla_{x} u\right\|_{2}^{2}+\left\|\nabla_{x} v\right\|_{2}^{2}\right) \\
& +c_{\sigma}\left(1+\alpha\|\rho\|_{L^{n / 2}\left(\mathbb{R}^{n}\right)}^{2}\right)\left(\left(g_{1} \circ \nabla_{x} u\right)+\left(g_{2} \circ \nabla_{x} v\right)\right) \\
& -c_{\sigma}\|\rho\|_{L^{s}\left(\mathbb{R}^{n}\right)}^{q}\left(\left(g_{1}^{\prime} \circ \nabla_{x} u\right)^{q / 2}+\left(g_{2}^{\prime} \circ \nabla_{x} v\right)^{q / 2}\right) \\
& +\left(\sigma-\int_{0}^{t} g(s) d s\right)\left(\left\|u^{\prime}\right\|_{L_{\rho}^{q}\left(\mathbb{R}^{n}\right)}^{q / 2}+\left\|v^{\prime}\right\|_{L_{\rho}^{q}\left(\mathbb{R}^{n}\right)}^{q / 2}\right) \\
& +m_{1} c_{3}\left(\left\|\nabla_{x} u\right\|_{2}^{2(\gamma+1)}+\left\|\nabla_{x} v\right\|_{2}^{2(\gamma+1)}\right) .
\end{aligned}
$$

where

$$
\int_{0}^{t} g(s) d s \leq \min \left\{\int_{0}^{t} g_{1}(s) d s, \int_{0}^{t} g_{2}(s) d s\right\}
$$


Proof. Exploiting Eq. (1.1), (3.5) to get

$$
\begin{aligned}
\psi_{2}^{\prime}(t) & =-\int_{\mathbb{R}^{n}} \rho(x)\left(\left|u^{\prime}\right|^{q-2} u^{\prime}\right)^{\prime} \int_{0}^{t} g_{1}(t-s)(u(t)-u(s)) d s d x \\
& -\int_{\mathbb{R}^{n}} \rho(x)\left|u^{\prime}\right|^{q-2} u^{\prime} \int_{0}^{t} g_{1}^{\prime}(t-s)(u(t)-u(s)) d s d x-\int_{0}^{t} g_{1}(s) d s\left\|u^{\prime}\right\|_{L_{\rho}^{q}\left(\mathbb{R}^{n}\right)}^{q} \\
& -\int_{\mathbb{R}^{n}} \rho(x)\left(\left|v^{\prime}\right|^{q-2} v^{\prime}\right)^{\prime} \int_{0}^{t} g_{2}(t-s)(v(t)-v(s)) d s d x \\
& -\int_{\mathbb{R}^{n}} \rho(x)\left|v^{\prime}\right|^{q-2} v^{\prime} \int_{0}^{t} g_{2}^{\prime}(t-s)(v(t)-v(s)) d s d x-\int_{0}^{t} g_{2}(s) d s\left\|v^{\prime}\right\|_{L_{\rho}^{q}}^{q}\left(\mathbb{R}^{n}\right) \\
& =\int_{\mathbb{R}^{n}} M\left(\|\nabla u\|_{2}^{2}\right) \nabla_{x} u \int_{0}^{t} g_{1}(t-s)\left(\nabla_{x} u(t)-\nabla_{x} u(s)\right) d s d x \\
& -\int_{\mathbb{R}^{n}}\left(\int_{0}^{t} g_{1}(t-s) \nabla_{x} u(s, x) d s\right)\left(\int_{0}^{t} g_{1}(t-s)\left(\nabla_{x} u(t)-\nabla_{x} u(s)\right) d s\right) d x \\
& -\int_{\mathbb{R}^{n}} \rho(x)\left|u^{\prime}\right|^{q-2} u^{\prime} \int_{0}^{t} g_{1}^{\prime}(t-s)(u(t)-u(s)) d s d x \\
& -\int_{0}^{t} g_{1}(s) d s\left\|u^{\prime}\right\|_{L_{\rho}^{q}\left(\mathbb{R}^{n}\right)}^{q}+\alpha \int_{\mathbb{R}^{n}} \rho(x) v \int_{0}^{t} g_{1}(t-s)(u(t)-u(s)) d s d x \\
& +\int_{\mathbb{R}^{n}} M\left(\|\nabla v\|_{2}^{2}\right) \nabla_{x} v \int_{0}^{t} g_{2}(t-s)\left(\nabla_{x} v(t)-\nabla_{x} v(s)\right) d s d x \\
& -\int_{\mathbb{R}^{n}}\left(\int_{0}^{t} g_{2}(t-s) \nabla_{x} v(s, x) d s\right)\left(\int_{0}^{t} g_{2}(t-s)\left(\nabla_{x} v(t)-\nabla_{x} v(s)\right) d s\right) d x \\
& -\int_{\mathbb{R}^{n}} \rho(x)\left|v^{\prime}\right|^{q-2} v^{\prime} \int_{0}^{t} g_{2}^{\prime}(t-s)(v(t)-v(s)) d s d x \\
& -\int_{0}^{t} g_{2}(s) d s\left\|v^{\prime}\right\|_{L_{\rho}^{q}\left(\mathbb{R}^{n}\right)}^{q}+\alpha \int_{\mathbb{R}^{n}} \rho(x) u \int_{0}^{t} g_{2}(t-s)(v(t)-v(s)) d s d x,
\end{aligned}
$$

then

$$
\begin{aligned}
\psi_{2}^{\prime}(t) & =\left(m_{0}-\int_{0}^{t} g_{1}(s) d s\right) \int_{\mathbb{R}^{n}} \nabla_{x} u \int_{0}^{t} g_{1}(t-s)\left(\nabla_{x} u(t)-\nabla_{x} u(s)\right) d s d x \\
& +\int_{\mathbb{R}^{n}}\left(\int_{0}^{t} g_{1}(t-s)\left(\nabla_{x} u(t)-\nabla_{x} u(s)\right) d s\right)^{2} d x+c_{1} m_{1}\left\|\nabla_{x} u\right\|_{2}^{2(\gamma+1)} \\
& -\int_{\mathbb{R}^{n}} \rho(x)\left|u^{\prime}\right|^{q-2} u^{\prime} \int_{0}^{t} g_{1}^{\prime}(t-s)(u(t)-u(s)) d s d x \\
& -\int_{0}^{t} g_{1}(s) d s\left\|u^{\prime}\right\|_{L_{\rho}^{q}\left(\mathbb{R}^{n}\right)}^{q}+c\left(g_{1} \circ \nabla_{x} u\right)(t) \\
& +\left(m_{0}-\int_{0}^{t} g_{2}(s) d s\right) \int_{\mathbb{R}^{n}} \nabla_{x} v \int_{0}^{t} g_{2}(t-s)\left(\nabla_{x} v(t)-\nabla_{x} v(s)\right) d s d x \\
& +\int_{\mathbb{R}^{n}}\left(\int_{0}^{t} g_{2}(t-s)\left(\nabla_{x} v(t)-\nabla_{x} v(s)\right) d s\right)^{2} d x+c_{2} m_{1}\left\|\nabla_{x} v\right\|_{2}^{2(\gamma+1)}
\end{aligned}
$$




$$
\begin{aligned}
& -\quad \int_{\mathbb{R}^{n}} \rho(x)\left|v^{\prime}\right|^{q-2} v^{\prime} \int_{0}^{t} g_{2}^{\prime}(t-s)(v(t)-v(s)) d s d x \\
& -\quad \int_{0}^{t} g_{2}(s) d s\left\|v^{\prime}\right\|_{L_{\rho}^{q}\left(\mathbb{R}^{n}\right)}^{q}+c\left(g_{2} \circ \nabla_{x} v\right)(t) \\
& +\alpha \int_{\mathbb{R}^{n}} \rho(x)\left(v \int_{0}^{t} g_{1}(t-s)(u(t)-u(s)) d s+u \int_{0}^{t} g_{2}(t-s)(v(t)-v(s)) d s\right) d x
\end{aligned}
$$

By Holder's and Young's inequalities and Lemma 3.1, we estimate the last term as

$$
\begin{aligned}
& \alpha \int_{\mathbb{R}^{n}} \rho(x) v \int_{0}^{t} g_{1}(t-s)(u(t)-u(s)) d s d x \\
& \leq \alpha\left(\int_{\mathbb{R}^{n}} \rho(x)|v|^{2} d x\right)^{1 / 2} \times \\
& \left(\int_{\mathbb{R}^{n}} \rho(x)\left|\int_{0}^{t} g_{1}(t-s)(u(t)-u(s)) d s\right|^{2}\right)^{1 / 2} \\
& \leq \alpha \sigma\|v\|_{L_{\rho}^{2}\left(\mathbb{R}^{n}\right)}^{2}+\alpha c_{\sigma}\left\|\int_{0}^{t} g_{1}(t-s)(u(t)-u(s)) d s\right\|_{L_{\rho}^{2}\left(\mathbb{R}^{n}\right)}^{2} \\
& \leq \alpha \sigma\|\rho\|_{L^{n / 2}\left(\mathbb{R}^{n}\right)}^{2}\left\|\nabla_{x} v\right\|_{2}^{2}+\alpha c_{\sigma}\|\rho\|_{L^{n / 2}\left(\mathbb{R}^{n}\right)}^{2}\left(g_{1} \circ \nabla_{x} u\right)(t) .
\end{aligned}
$$

and

$$
\begin{aligned}
& \alpha \int_{\mathbb{R}^{n}} \rho(x) u \int_{0}^{t} g_{2}(t-s)(v(t)-v(s)) d s d x \\
& \leq \alpha\left(\int_{\mathbb{R}^{n}} \rho(x)|u|^{2} d x\right)^{1 / 2} \times \\
& \left(\int_{\mathbb{R}^{n}} \rho(x)\left|\int_{0}^{t} g_{2}(t-s)(v(t)-v(s)) d s\right|^{2}\right)^{1 / 2} \\
& \leq \alpha \sigma\|u\|_{L_{\rho}^{2}\left(\mathbb{R}^{n}\right)}^{2}+\alpha c_{\sigma}\left\|\int_{0}^{t} g_{2}(t-s)(v(t)-v(s)) d s\right\|_{L_{\rho}^{2}\left(\mathbb{R}^{n}\right)}^{2} \\
& \leq \alpha \sigma\|\rho\|_{L^{n / 2}\left(\mathbb{R}^{n}\right)}^{2}\left\|\nabla_{x} u\right\|_{2}^{2}+\alpha c_{\sigma}\|\rho\|_{L^{n / 2}\left(\mathbb{R}^{n}\right)}^{2}\left(g_{2} \circ \nabla_{x} v\right)(t) .
\end{aligned}
$$

and for the exponents $\frac{q}{q-1}, q$ 


$$
\begin{aligned}
& -\int_{\mathbb{R}^{n}} \rho(x)\left|u^{\prime}\right|^{q-2} u^{\prime} \int_{0}^{t} g_{1}^{\prime}(t-s)(u(t)-u(s)) d s d x \\
& \leq\left(\int_{\mathbb{R}^{n}} \rho(x)\left|u^{\prime}\right|^{q} d x\right)^{(q-1) / q} \times \\
& \left(\int_{\mathbb{R}^{n}} \rho(x)\left|\int_{0}^{t} g_{1}^{\prime}(t-s)(u(t)-u(s)) d s\right|^{q}\right)^{1 / q} \\
& \leq \sigma\left\|u^{\prime}\right\|_{L_{\rho}^{q}\left(\mathbb{R}^{n}\right)}^{q}+c_{\sigma}\left\|\int_{0}^{t}-g_{1}^{\prime}(t-s)(u(t)-u(s)) d s\right\|_{L_{\rho}^{q}\left(\mathbb{R}^{n}\right)}^{q} \\
& \leq \sigma\left\|u^{\prime}\right\|_{L_{\rho}^{q}\left(\mathbb{R}^{n}\right)}^{q}-c_{\sigma}\|\rho\|_{L^{s}\left(\mathbb{R}^{n}\right)}^{q}\left(g_{1}^{\prime} \circ \nabla_{x} u\right)^{q / 2}(t) .
\end{aligned}
$$

and

$$
\begin{aligned}
& -\int_{\mathbb{R}^{n}} \rho(x)\left|v^{\prime}\right|^{q-2} v^{\prime} \int_{0}^{t} g_{2}^{\prime}(t-s)(v(t)-v(s)) d s d x \\
& \leq\left(\int_{\mathbb{R}^{n}} \rho(x)\left|v^{\prime}\right|^{q} d x\right)^{(q-1) / q} \times \\
& \left(\int_{\mathbb{R}^{n}} \rho(x)\left|\int_{0}^{t} g_{2}^{\prime}(t-s)(v(t)-v(s)) d s\right|^{q}\right)^{1 / q} \\
& \leq \sigma\left\|v^{\prime}\right\|_{L_{\rho}^{q}\left(\mathbb{R}^{n}\right)}^{q}+c_{\sigma}\left\|\int_{0}^{t}-g_{2}^{\prime}(t-s)(v(t)-v(s)) d s\right\|_{L_{\rho}^{q}\left(\mathbb{R}^{n}\right)}^{q} \\
& \leq \sigma\left\|v^{\prime}\right\|_{L_{\rho}^{q}\left(\mathbb{R}^{n}\right)}^{q}-c_{\sigma}\|\rho\|_{L^{s}\left(\mathbb{R}^{n}\right)}^{q}\left(g_{2}{ }^{\prime} \circ \nabla_{x} v\right)^{q / 2}(t) .
\end{aligned}
$$

Using Young's and Poincare's inequalities and Lemma 2.1 for $\theta=1 / 2$, we obtain

$$
\begin{aligned}
\psi_{2}^{\prime}(t) & \leq \sigma\left(1+\alpha\|\rho\|_{L^{n / 2}\left(\mathbb{R}^{n}\right)}^{2}\right)\left(\left\|\nabla_{x} u\right\|_{2}^{2}+\left\|\nabla_{x} v\right\|_{2}^{2}\right) \\
& +c_{\sigma}\left(1+\alpha\|\rho\|_{L^{n / 2}\left(\mathbb{R}^{n}\right)}^{2}\right)\left(\left(g_{1} \circ \nabla_{x} u\right)+\left(g_{2} \circ \nabla_{x} v\right)\right) \\
& -c_{\sigma}\|\rho\|_{L^{s}\left(\mathbb{R}^{n}\right)}^{q}\left(\left(g_{1}^{\prime} \circ \nabla_{x} u\right)^{q / 2}+\left(g_{2}^{\prime} \circ \nabla_{x} v\right)^{q / 2}\right) \\
& +\left(\sigma-\int_{0}^{t} g_{1}(s) d s\right)\left\|u^{\prime}\right\|_{L_{\rho}^{q}\left(\mathbb{R}^{n}\right)}^{q / 2}+\left(\sigma-\int_{0}^{t} g_{2}(s) d s\right)\left\|v^{\prime}\right\|_{L_{\rho}^{q}\left(\mathbb{R}^{n}\right)}^{q / 2} \\
& +m_{1}\left(c_{1}\left\|\nabla_{x} u\right\|_{2}^{2(\gamma+1)}+c_{2}\left\|\nabla_{x} v\right\|_{2}^{2(\gamma+1)}\right) .
\end{aligned}
$$

We need the next Lemma, which means that there is equivalent between the Lyapunov and energy functions, that is for $\xi_{1}, \xi_{2}>1$, we have

$$
\beta_{1} L(t) \leq E(t) \leq \beta_{2} L(t)
$$

holds for two positive constants $\beta_{1}$ and $\beta_{2}$. 
Lemma 3.4. For $\xi_{1}, \xi_{2}>1$, we have

$$
L(t) \sim E(t)
$$

Proof. By (3.3) we have

$$
\begin{aligned}
\left|L(t)-\xi_{1} E(t)\right| & \leq\left|\psi_{1}(t)\right|+\xi_{2}\left|\psi_{2}(t)\right| \\
& \leq\left.\int_{\mathbb{R}^{n}}|\rho(x) u| u^{\prime}\right|^{q-2} u^{\prime}\left|d x+\int_{\mathbb{R}^{n}}\right| \rho(x) v\left|v^{\prime}\right|^{q-2} v^{\prime} \mid d x \\
& +\left.\xi_{2} \int_{\mathbb{R}^{n}}|\rho(x)| u^{\prime}\right|^{q-2} u^{\prime} \int_{0}^{t} g_{1}(t-s)(u(t)-u(s)) d s \mid d x \\
& +\left.\xi_{2} \int_{\mathbb{R}^{n}}|\rho(x)| v^{\prime}\right|^{q-2} v^{\prime} \int_{0}^{t} g_{2}(t-s)(v(t)-v(s)) d s \mid d x .
\end{aligned}
$$

Thanks to Holder and Young's inequalities with exponents $\frac{q}{q-1}, q$, since $\frac{2 n}{n+2} \geq q \geq 2$, we have by using Lemma 3.1

$$
\begin{aligned}
\left.\int_{\mathbb{R}^{n}}|\rho(x) u| u^{\prime}\right|^{q-2} u^{\prime} \mid d x & \leq\left(\int_{\mathbb{R}^{n}} \rho(x)|u|^{q} d x\right)^{1 / q}\left(\int_{\mathbb{R}^{n}} \rho(x)\left|u^{\prime}\right|^{q} d x\right)^{(q-1) / q} \\
& \leq \frac{1}{q}\left(\int_{\mathbb{R}^{n}} \rho(x)|u|^{q} d x\right)+\frac{q-1}{q}\left(\int_{\mathbb{R}^{n}} \rho(x)\left|u^{\prime}\right|^{q} d x\right) \\
& \leq c\left\|u^{\prime}\right\|_{L_{\rho}^{q}\left(\mathbb{R}^{n}\right)}^{q}+c\|\rho\|_{L^{s}\left(\mathbb{R}^{n}\right)}^{q}\left\|\nabla_{x} u\right\|_{2}^{q}
\end{aligned}
$$

and

$$
\begin{aligned}
\left.\int_{\mathbb{R}^{n}}|\rho(x) v| v^{\prime}\right|^{q-2} v^{\prime} \mid d x & \leq\left(\int_{\mathbb{R}^{n}} \rho(x)|v|^{q} d x\right)^{1 / q}\left(\int_{\mathbb{R}^{n}} \rho(x)\left|v^{\prime}\right|^{q} d x\right)^{(q-1) / q} \\
& \leq \frac{1}{q}\left(\int_{\mathbb{R}^{n}} \rho(x)|v|^{q} d x\right)+\frac{q-1}{q}\left(\int_{\mathbb{R}^{n}} \rho(x)\left|v^{\prime}\right|^{q} d x\right) \\
& \leq c\left\|v^{\prime}\right\|_{L_{\rho}^{q}\left(\mathbb{R}^{n}\right)}^{q}+c\|\rho\|_{L^{s}\left(\mathbb{R}^{n}\right)}^{q}\left\|\nabla_{x} v\right\|_{2}^{q}
\end{aligned}
$$

and

$$
\begin{aligned}
& \int_{\mathbb{R}^{n}}\left|\left(\rho(x)^{\frac{q-1}{q}}\left|u^{\prime}\right|^{q-2} u^{\prime}\right)\left(\rho(x)^{\frac{1}{q}} \int_{0}^{t} g_{1}(t-s)(u(t)-u(s)) d s\right)\right| d x \\
& \leq\left(\int_{\mathbb{R}^{n}} \rho(x)\left|u^{\prime}\right|^{q} d x\right)^{(q-1) / q} \times \\
& \left(\int_{\mathbb{R}^{n}} \rho(x)\left|\int_{0}^{t} g_{1}(t-s)(u(t)-u(s)) d s\right|^{q} d x\right)^{1 / q} \\
& \leq \frac{q-1}{q}\left\|u^{\prime}\right\|_{L_{\rho}^{q}\left(\mathbb{R}^{n}\right)}^{q}+\frac{1}{q}\left\|\int_{0}^{t} g_{1}(t-s)(u(t)-u(s)) d s\right\|_{L_{\rho}^{q}\left(\mathbb{R}^{n}\right)}^{q} \\
& \leq \frac{q-1}{q}\left\|u^{\prime}\right\|_{L_{\rho}^{q}\left(\mathbb{R}^{n}\right)}^{q}+\frac{1}{q}\|\rho\|_{L^{s}\left(\mathbb{R}^{n}\right)}^{q}\left(g_{1} \circ \nabla_{x} u\right)^{q / 2}(t) .
\end{aligned}
$$


and

$$
\begin{aligned}
& \int_{\mathbb{R}^{n}}\left|\left(\rho(x)^{\frac{q-1}{q}}\left|v^{\prime}\right|^{q-2} v^{\prime}\right)\left(\rho(x)^{\frac{1}{q}} \int_{0}^{t} g_{2}(t-s)(v(t)-v(s)) d s\right)\right| d x \\
& \leq\left(\int_{\mathbb{R}^{n}} \rho(x)\left|v^{\prime}\right|^{q} d x\right)^{(q-1) / q} \times \\
& \left(\int_{\mathbb{R}^{n}} \rho(x)\left|\int_{0}^{t} g_{2}(t-s)(v(t)-v(s)) d s\right|^{q} d x\right)^{1 / q} \\
& \leq \frac{q-1}{q}\left\|v^{\prime}\right\|_{L_{\rho}^{q}\left(\mathbb{R}^{n}\right)}^{q}+\frac{1}{q}\left\|\int_{0}^{t} g_{2}(t-s)(v(t)-v(s)) d s\right\|_{L_{\rho}^{q}\left(\mathbb{R}^{n}\right)}^{q} \\
& \leq \frac{q-1}{q}\left\|v^{\prime}\right\|_{L_{\rho}^{q}\left(\mathbb{R}^{n}\right)}^{q}+\frac{1}{q}\|\rho\|_{L^{s}\left(\mathbb{R}^{n}\right)}^{q}\left(g_{2} \circ \nabla_{x} v\right)^{q / 2}(t) .
\end{aligned}
$$

Then, since $q \geq 2$, we have

$$
\begin{aligned}
\left|L(t)-\xi_{1} E(t)\right| & \leq c\left(E(t)+E^{q / 2}(t)\right) \\
& \leq c\left(E(t)+E(t) E^{(q / 2)-1}(t)\right) \\
& \leq c\left(E(t)+E(t) E^{(q / 2)-1}(0)\right) \\
& \leq c E(t) .
\end{aligned}
$$

Therefore, we can choose $\xi_{1}$ so that

$$
L(t) \sim E(t) .
$$

Proof of Theorem 3.1 From (2.12), results of Lemma 3.2 and Lemma 3.3, we have

$$
\begin{aligned}
L^{\prime}(t) & =\xi_{1} E^{\prime}(t)+\psi_{1}^{\prime}(t)+\xi_{2} \psi_{2}^{\prime}(t) \\
& \leq\left(\frac{\xi_{1}}{2}-c_{\sigma} \xi_{2}\|\rho\|_{L^{s}\left(\mathbb{R}^{n}\right)}^{q}\right)\left[\left(g_{1}^{\prime} \circ \nabla_{x} u\right)^{q / 2}+\left(g_{2}^{\prime} \circ \nabla_{x} v\right)^{q / 2}\right] \\
& +M_{0}\left[\left(g_{2} \circ \nabla_{x} u\right)+\left(g_{2} \circ \nabla_{x} v\right)\right]-M_{1}\left[\left\|u^{\prime}\right\|_{L_{\rho}^{q}\left(\mathbb{R}^{n}\right)}^{q}+\left\|v^{\prime}\right\|_{L_{\rho}^{q}\left(\mathbb{R}^{n}\right)}^{q}\right] \\
& +c m_{1}\left(1+\xi_{2}\right)\left[\left\|\nabla_{x} u\right\|_{2}^{2(\gamma+1)}+\left\|\nabla_{x} v\right\|_{2}^{2(\gamma+1)}\right]-M_{2}\left[\left\|\nabla_{x} u\right\|_{2}^{2}+\left\|\nabla_{x} v\right\|_{2}^{2}\right]
\end{aligned}
$$

where

$$
\begin{gathered}
M_{0}=\left(\frac{4 \xi_{2} c\left(1+\alpha\|\rho\|_{L^{n / 2}\left(\mathbb{R}^{n}\right)}^{2}\right)+(1-l)}{4 \sigma}\right), \\
M_{1}=\left(\xi_{2}\left(\int_{0}^{t_{1}} g(s) d s-\sigma\right)+c|\alpha|\|\rho\|_{L^{n / 2}\left(\mathbb{R}^{n}\right)}^{-1}-1\right), \\
M_{2}=\left(-\xi_{2} \sigma\left(1+\alpha\|\rho\|_{L^{n / 2}\left(\mathbb{R}^{n}\right)}^{2}\right)+(l-\sigma)\right),
\end{gathered}
$$


and $t_{1}$ was introduced in Remark 2.1.

We choose $\sigma$ so small that $\xi_{1}>2 c_{\sigma}\|\rho\|_{L^{s}\left(\mathbb{R}^{n}\right)}^{q} \xi_{2}$. Whence $\sigma$ is fixed, we can choose $\xi_{1}, \xi_{2}$ large enough so that $M_{1}, M_{2}>0$, which yields

$$
\begin{aligned}
L^{\prime}(t) & \leq M_{0}\left[\left(g_{1} \circ \nabla_{x} u\right)+\left(g_{2} \circ \nabla_{x} v\right)\right] \\
& +c m_{1}\left[\left\|\nabla_{x} u\right\|_{2}^{2(\gamma+1)}+\left\|\nabla_{x} v\right\|_{2}^{2(\gamma+1)}\right]-c E(t), \quad \text { for all } \quad t \geq t_{1}
\end{aligned}
$$

Now we set $F(t)=L(t)+c E(t)$, which is equivalent to $E(t)$. Then by (3.13), we get for a positive constant $c$

(3.14) $F^{\prime}(t)=L^{\prime}(t)+c E^{\prime}(t)$

$$
\begin{aligned}
& \leq-c E(t)+c \int_{\mathbb{R}^{n}} \int_{t_{1}}^{t} g_{1}(t-s)\left|\nabla_{x} u(t)-\nabla_{x} u(s)\right|^{2} d s d x \\
& +c \int_{\mathbb{R}^{n}} \int_{t_{1}}^{t} g_{2}(t-s)\left|\nabla_{x} v(t)-\nabla_{x} v(s)\right|^{2} d s d x, \text { for all } t \geq t_{1} .
\end{aligned}
$$

By (2.4) and (2.12), we have for all $t \geq t_{1}$

$$
\begin{aligned}
& \int_{\mathbb{R}^{n}} \int_{0}^{t_{1}} g_{1}(t-s)\left|\nabla_{x} u(t)-\nabla_{x} u(s)\right|^{2} d s d x+\int_{\mathbb{R}^{n}} \int_{0}^{t_{1}} g_{2}(t-s)\left|\nabla_{x} v(t)-\nabla_{x} v(s)\right|^{2} d s d x \\
& \leq-\frac{1}{k}\left(\int_{\mathbb{R}^{n}} \int_{0}^{t_{1}} g_{1}^{\prime}(t-s)\left|\nabla_{x} u(t)-\nabla_{x} u(s)\right|^{2} d s d x+\int_{\mathbb{R}^{n}} \int_{0}^{t_{1}} g_{2}^{\prime}(t-s)\left|\nabla_{x} v(t)-\nabla_{x} v(s)\right|^{2} d s d x\right) \\
& \leq-c E^{\prime}(t) .
\end{aligned}
$$

At this point, we define

$$
\begin{aligned}
I(t) & =\int_{t_{1}}^{t} H_{0}\left(-g_{1}^{\prime}(s)\right)\left(g_{1} \circ \nabla_{x} u\right)(t) d s \\
& +\int_{t_{1}}^{t} H_{0}\left(-g_{2}^{\prime}(s)\right)\left(g_{2} \circ \nabla_{x} v\right)(t) d s .
\end{aligned}
$$

Since $\int_{0}^{+\infty} H_{0}\left(-g_{i}^{\prime}(s)\right) g(s) d s<+\infty, i=1,2$, from (2.12) we have

$$
\begin{aligned}
& I(t)=\int_{t_{1}}^{t} H_{0}\left(-g_{1}^{\prime}(s)\right) \int_{\mathbb{R}^{n}} g_{1}(s)\left|\nabla_{x} u(t)-\nabla_{x} u(t-s)\right|^{2} d x d s \\
& +\int_{t_{1}}^{t} H_{0}\left(-g_{2}^{\prime}(s)\right) \int_{\mathbb{R}^{n}} g_{2}(s)\left|\nabla_{x} v(t)-\nabla_{x} v(t-s)\right|^{2} d x d s \\
& \leq 2 \int_{t_{1}}^{t} H_{0}\left(-g_{1}^{\prime}(s)\right) g_{1}(s) \int_{\mathbb{R}^{n}}\left|\nabla_{x} u(t)\right|^{2}+\left|\nabla_{x} u(t-s)\right|^{2} d x d s \\
& +2 \int_{t_{1}}^{t} H_{0}\left(-g_{2}^{\prime}(s)\right) g_{2}(s) \int_{\mathbb{R}^{n}}\left|\nabla_{x} v(t)\right|^{2}+\left|\nabla_{x} v(t-s)\right|^{2} d x d s \\
& \leq c E(0)\left[\int_{t_{1}}^{t} H_{0}\left(-g_{1}^{\prime}(s)\right) g_{1}(s) d s+\int_{t_{1}}^{t} H_{0}\left(-g_{2}^{\prime}(s)\right) g_{2}(s) d s\right] .
\end{aligned}
$$


As in ([16], Eq. (3.11)), we have $I(t)<1$. Now, we define again a new functional $\lambda(t)$ related to $I(t)$ as

$$
\begin{aligned}
\lambda(t) & =-\int_{t_{1}}^{t} H_{0}\left(-g_{1}^{\prime}(s)\right) g_{1}^{\prime}(s) \int_{\mathbb{R}^{n}} g_{1}(s)\left|\nabla_{x} u(t)-\nabla_{x} u(t-s)\right|^{2} d x d s \\
& -\int_{t_{1}}^{t} H_{0}\left(-g_{2}^{\prime}(s)\right) g_{2}^{\prime}(s) \int_{\mathbb{R}^{n}} g_{2}(s)\left|\nabla_{x} v(t)-\nabla_{x} v(t-s)\right|^{2} d x d s .
\end{aligned}
$$

From (A1)-(A2) and Remark 2.1 we get

$$
H_{0}\left(-g_{i}^{\prime}(s)\right) g_{i}(s) \leq H_{0}\left(H\left(g_{i}(s)\right)\right) g_{i}(s)=D\left(g_{i}(s)\right) g_{i}(s) \leq k_{0} .
$$

for some positive constant $k_{0}$. Then, for all $t \geq t_{1}$

$$
\begin{aligned}
\lambda(t) & \leq-k_{0} \int_{t_{1}}^{t} g_{1}^{\prime}(s) \int_{\mathbb{R}^{n}}\left|\nabla_{x} u(t)-\nabla_{x} u(t-s)\right|^{2} d x d s \\
& -k_{0} \int_{t_{1}}^{t} g_{2}^{\prime}(s) \int_{\mathbb{R}^{n}}\left|\nabla_{x} v(t)-\nabla_{x} v(t-s)\right|^{2} d x d s \\
& \leq-k_{0} \int_{t_{1}}^{t} g_{1}^{\prime}(s) \int_{\mathbb{R}^{n}}\left|\nabla_{x} u(t)\right|^{2}+\left|\nabla_{x} u(t-s)\right|^{2} d x d s \\
& -k_{0} \int_{t_{1}}^{t} g_{2}^{\prime}(s) \int_{\mathbb{R}^{n}}\left|\nabla_{x} v(t)\right|^{2}+\left|\nabla_{x} v(t-s)\right|^{2} d x d s \\
& \leq-c E(0)\left[\int_{t_{1}}^{t} g_{1}^{\prime}(s) d s+\int_{t_{1}}^{t} g_{2}^{\prime}(s) d s\right] \\
& \leq c E(0) \max \left\{g_{1}\left(t_{1}\right), g_{2}\left(t_{1}\right)\right\} \\
& <\min \left\{r, H(r), H_{0}(r)\right\} .
\end{aligned}
$$

Using the properties of $H_{0}$ (strictly convex in $(0, r], H_{0}(0)=0$ ), then for $x \in$ $(0, r], \theta \in[0,1]$

$$
H_{0}(\theta x) \leq \theta H_{0}(x) .
$$

Using Remark 2.1, (3.16), (3.18) and Jensen's inequality leads to 


$$
\begin{aligned}
\lambda(t) & =I^{-1}(t)\left\{\int_{t_{1}}^{t} I(t) H_{0}\left[H_{0}^{-1}\left(-g_{1}^{\prime}(s)\right)\right] H_{0}\left(-g_{1}^{\prime}(s)\right) g_{1}^{\prime}(s) \int_{\mathbb{R}^{n}} g_{1}(s)\left|\nabla_{x} u(t)-\nabla_{x} u(t-s)\right|^{2} d x d s\right. \\
& \left.+\int_{t_{1}}^{t} I(t) H_{0}\left[H_{0}^{-1}\left(-g_{2}^{\prime}(s)\right)\right] H_{0}\left(-g_{2}^{\prime}(s)\right) g_{2}^{\prime}(s) \int_{\mathbb{R}^{n}} g_{2}(s)\left|\nabla_{x} v(t)-\nabla_{x} v(t-s)\right|^{2} d x d s\right\} \\
& \geq I^{-1}(t)\left\{\int_{t_{1}}^{t} H_{0}\left[I(t) H_{0}^{-1}\left(-g_{1}^{\prime}(s)\right)\right] H_{0}\left(-g_{1}^{\prime}(s)\right) g_{1}^{\prime}(s) \int_{\mathbb{R}^{n}} g_{1}(s)\left|\nabla_{x} u(t)-\nabla_{x} u(t-s)\right|^{2} d x d s\right. \\
& \left.+\int_{t_{1}}^{t} H_{0}\left[I(t) H_{0}^{-1}\left(-g_{2}^{\prime}(s)\right)\right] H_{0}\left(-g_{2}^{\prime}(s)\right) g_{2}^{\prime}(s) \int_{\mathbb{R}^{n}} g_{2}(s)\left|\nabla_{x} v(t)-\nabla_{x} v(t-s)\right|^{2} d x d s\right\} \\
& \geq H_{0}\left(I^{-1}(t) \int_{t_{1}}^{t} I(t) H_{0}^{-1}\left(-g_{1}^{\prime}(s)\right) H_{0}\left(-g_{1}^{\prime}(s)\right) g_{1}^{\prime}(s) \int_{\mathbb{R}^{n}} g_{1}(s)\left|\nabla_{x} u(t)-\nabla_{x} u(t-s)\right|^{2} d x d s\right. \\
& \left.+I^{-1}(t) \int_{t_{1}}^{t} I(t) H_{0}^{-1}\left(-g_{2}^{\prime}(s)\right) H_{0}\left(-g_{2}^{\prime}(s)\right) g_{2}^{\prime}(s) \int_{\mathbb{R}^{n}} g_{2}(s)\left|\nabla_{x} v(t)-\nabla_{x} v(t-s)\right|^{2} d x d s\right) \\
& \geq H_{0}\left(\int_{t_{1}}^{t} \int_{\mathbb{R}^{n}} g_{1}(s)\left|\nabla_{x} u(t)-\nabla_{x} u(t-s)\right|^{2} d x d s+\int_{t_{1}}^{t} \int_{\mathbb{R}^{n}} g_{2}(s)\left|\nabla_{x} v(t)-\nabla_{x} v(t-s)\right|^{2} d x d s\right)
\end{aligned}
$$

which implies

$$
\begin{aligned}
& \int_{t_{1}}^{t} \int_{\mathbb{R}^{n}} g_{1}(s)\left|\nabla_{x} u(t)-\nabla_{x} u(t-s)\right|^{2} d x d s+\int_{t_{1}}^{t} \int_{\mathbb{R}^{n}} g_{2}(s)\left|\nabla_{x} v(t)-\nabla_{x} v(t-s)\right|^{2} d x d s \\
& \leq H_{0}^{-1}(\lambda(t)) .
\end{aligned}
$$

Then

$$
F^{\prime}(t) \leq-c E(t)+c H_{0}^{-1}(\lambda(t)), \text { for all } t \geq t_{1} .
$$

Now, we will following the steps in $([16])$ and using the fact that $E^{\prime} \leq 0,0<H_{0}^{\prime}, 0<$ $H_{0}^{\prime \prime}$ on $(0, r]$ to define the functional

$$
F_{1}(t)=H_{0}^{\prime}\left(a \frac{E(t)}{E(0)}\right) F(t)+c E(t), \quad a<r, 0<c,
$$

where $F_{1}(t) \sim E(t)$ and

$$
\begin{aligned}
F_{1}^{\prime}(t) & =a \frac{E^{\prime}(t)}{E(0)} H_{0}^{\prime \prime}\left(a \frac{E(t)}{E(0)}\right) F(t)+H_{0}^{\prime}\left(a \frac{E(t)}{E(0)}\right) F^{\prime}(t)+c E^{\prime}(t) \\
& \leq-c E(t) H_{0}^{\prime}\left(a \frac{E(t)}{E(0)}\right)+c H_{0}^{\prime}\left(a \frac{E(t)}{E(0)}\right) H_{0}^{-1}(\lambda(t))+c E^{\prime}(t) .
\end{aligned}
$$

Let $H_{0}^{*}$ given in Remark 2.1 and using Young's inequality (2.5) with $A=H_{0}^{\prime}\left(a \frac{E(t)}{E(0)}\right)$, $B=H_{0}^{-1}(\lambda(t))$, to get

$$
\begin{aligned}
F_{1}^{\prime}(t) & \leq-c E(t) H_{0}^{\prime}\left(a \frac{E(t)}{E(0)}\right)+c H_{0}^{*}\left(H_{0}^{\prime}\left(a \frac{E(t)}{E(0)}\right)\right)+c \lambda(t)+c E^{\prime}(t) \\
& \leq-c E(t) H_{0}^{\prime}\left(a \frac{E(t)}{E(0)}\right)+c a \frac{E(t)}{E(0)} H_{0}^{\prime}\left(a \frac{E(t)}{E(0)}\right)-c^{\prime} E^{\prime}(t)+c E^{\prime}(t)
\end{aligned}
$$


Choosing $a, c, c^{\prime}$, such that for all $t \geq t_{1}$ we have

$$
\begin{aligned}
F_{1}^{\prime}(t) & \leq-k \frac{E(t)}{E(0)} H_{0}^{\prime}\left(a \frac{E(t)}{E(0)}\right) \\
& =-k H_{2}\left(\frac{E(t)}{E(0)}\right),
\end{aligned}
$$

where $H_{2}(t)=t H_{0}^{\prime}\left(\alpha_{0} t\right)$. Using the strict convexity of $H_{0}$ on $(0, r]$, to find that $H_{2}^{\prime}, H_{2}$ are strict positives on $(0,1]$, then

$$
R(t)=\tau \frac{k_{1} F_{1}(t)}{E(0)} \sim E(t), \quad \tau \in(0,1)
$$

and

$$
R^{\prime}(t) \leq-\tau k_{0} H_{2}(R(t)), \quad k_{0} \in(0,+\infty), t \geq t_{1} .
$$

Then, a simple integration and a suitable choice of $\tau$ yield,

$$
R(t) \leq H_{1}^{-1}(b t+c), \quad b, c \in(0,+\infty), t \geq t_{1} .
$$

here $H_{1}(t)=\int_{t}^{1} H_{2}^{-1}(s) d s$. From (3.19), for a positive constant $\alpha_{3}$, we have

$$
E(t) \leq d H_{1}^{-1}(b t+c)
$$

The fact that $H_{1}$ is strictly decreasing function on $(0,1]$ and due to properties of $\mathrm{H}_{2}$, we have

$$
\lim _{t \rightarrow 0} H_{1}(t)=+\infty
$$

Then

$$
E(t) \leq d H_{1}^{-1}(b t+c), \quad \text { for all } t \geq 0
$$

This completes the proof of Theorem 3.1.

\section{RE F E R E N C E S}

1. M. Abdelli And A. Benaissa, Energy decay of solutions of degenerate Kirchhoff equation with a weak nonlinear dissipation, Nonlinear Anal, 69 (2008) ,1999-2008.

2. F. Alabau-Boussouira, F. And P. Cannarsa, A general method for proving sharp energy decay rates for memory-dissipative evolution equations, C. R. Math. Acad. Sci. Paris, Ser. I 347, (2009), 867-872.

3. V. I. ARnold, Mathematical Methods of Classical Mechanics, Springer-Verlag, New York, 1989

4. A. Benaissa, S. Mokeddem, Global existence and energy decay of solutions to the Cauchy problem for a wave equation with a weakly nonlinear dissipation, Abstr. Appl. Anal, 11(2004) 935-955. 
5. K. J. Brown, ; N. M. Stavrakakis, Global bifurcation results for semilinear elliptic equations on all of $\mathbb{R}^{n}$, Duke Math. J. 85 (1996), 77-94.

6. M.M. Cavalcanti, H.P. OQUendo, Frictional versus viscoelastic damping in a semilinear wave equation, SIAM J. Control Optim. 42(4)(2003)1310-1324.

7. C. M. Dafermos, H.P. Oquendo, Asymptotic stability in viscoelasticity, Arch. Ration. Mech. Anal. 37(1970), 297-308.

8. I. Lasiecka, S. A. Messaoudi And M. I. Mustafa, Note on intrinsic decay rates for abstract wave equations with memory, J. Math. Phys. 031504 (2013).

9. M. KAFINI, uniforme decay of solutions to Cauchy viscoelastic problems with density, Elecron. J. Differential Equations Vol.2011 (2011)No. 93, pp. 1-9.

10. M. Kafini And S. A. Messaoudi, On the uniform decay in viscoelastic problem in $\mathbb{R}^{n}$, Appl. Math. Comput 215 (2009) 1161-1169.

11. M. Kafini, S. A. Messaoudi and Nasser-eddine Tatar, Decay rate of solutions for a Cauchy viscoelastic evolution equation, Indag. Math. 22 (2011) 103-115.

12. N. I. KARAchalios, N.I; N.M. STAVRAKAKIS, Existence of global attractor for semilinear dissipative wave equations on $\mathbb{R}^{n}$, J. Differential Equations 157 (1999) 183-205.

13. G. Kirchioff, Vorlesungen uber Mechanik,3rd ed., Teubner, Leipzig, (1983).

14. P. Martinez, A new method to obtain decay rate estimates for dissipative systems, ESAIM Control Optim. Calc. Var. 4(1999)419-444.

15. S. A. Messaoudi And NAsser-Eddine TATAR, Uniform stabilization of solutions of a nonlinear system of viscoelastic equations, App. Anal. 87 (2008) 247-263.

16. Muhammad I. Mustafa And S. A. Messaoudi, General stability result for viscoelastic wave equations, J. Math. Phys. 53, 053702 (2012).

17. J. E. Munoz Rivera, Global solution on a quasilinear wave equation with memory, Boll. Unione Mat. Ital. B (7) 8 (1994), no. 2, 289-303.

18. Papadopulos, P.G. Stavrakakies, Global existence and blow-up results for an equations of Kirchhoff type on $\mathbb{R}^{n}$, Topol. Methods Nolinear Anal. 17, (2001), 91-109.

19. M. L. SAntos, Decay rates for solutions of a system of wave equations with memory, Elec. J. Diff. Equ. 38 (2002), 1-17.

20. R. Torrejon And J. M. Yong, On a quasilinear wave equation with memory, Nonlinear Anal. 16 (1991), no. 1, 61-78.

21. KH. ZenniR, General decay of solutions for damped wave equation of Kirchhoff type with density in $\mathbb{R}^{n}$, Ann Univ Ferrara. DOI 10.1007/s11565-015-0223-x (2015).

22. Y. ZHOU, A blow-up result for a nonlinear wave equation with damping and vanishing initial energy in $\mathbb{R}^{n}$, Appl. Math. Lett. 18 (2005), 281-286.

\author{
Abbes Benaissa \\ Laboratory of Analysis and Control of PDEs \\ Faculty of Exact Sciences \\ P. O. Box 89 \\ 22000 Sidi Bel Abbes, Algeria \\ benaissa_abbes@yahoo.com
}


Abderrahmane Beniani

Laboratory of Analysis and Control of PDEs

Faculty of Exact Sciences

P. O. Box 89

22000 Sidi Bel Abbes, Algeria

a.beniani@yahoo.fr

Khaled Zennir

College Of Sciences and Arts, Al-Ras,

Department of Mathematics

Al-Qassim, Kingdom Of Saudi Arabia

khaledzennir2@yahoo.com 